\title{
The Nursing of the Patients with Severe Craniocerebral Trauma Treated
}

\section{by Hyperbaric Oxygen}

\author{
Daoxin $J^{1}{ }^{1, a}$ Yanhua Guan ${ }^{1, b}$ \\ ${ }^{1}$ Luohe Medical College, Luohe City, Henan Province, China, 462000 \\ a email: \\ b email:
}

\begin{abstract}
Keywords: Hyperbaric Oxygen, Severe Craniocerebral Trauma, Clinical Treatment, Nursing Effects, Glasgow Coma Scale
\end{abstract}

\begin{abstract}
Craniocerebral trauma is a complex pathological variation, and it is a kind of energy metabolic dysfunction, which freely generates and increases microcirculation disturbance in vivo. Along with the increment of free radicals, the dysfunction of energy metabolism and microcirculation, the brain tissue showed various degrees of necrosis or degeneration. The disease has high morbidity, mortality and disability rates. The survivors mostly suffer from varied degrees of dysfunction and completely unable to take care of themselves, this increases burden to families and society. In recent years, hyperbaric oxygen has been applied some craniocerebral trauma patients, this method can increase the cure rate of craniocerebral trauma and decrease the disability rate of central nervous function, and also improves clinical therapeutic effect. The report shows that performing corresponding nursing measures can further enhance the cure rate during the treatment in patients with craniocerebral trauma, however the conclusion has not been confirmed. In order to explore the effects of clinical treatment and nursing of hyperbaric oxygen in severe craniocerebral trauma patients, 16 patients achieved good results after the surgery in the hospital from June 2013 to March 2016. The results as follows.
\end{abstract}

\section{Materials}

From June 2013 to March 2016, there were 16 patients, including 14 males and 2 females, have severe craniocerebral trauma surgery in the hospital . Their age between 3 to 55, and the average age is 28. And 4 patients have a coma when they first given treatment in hyperbaric oxygen chamber, 2 patients with tracheotomy and 11 patients with intubation (gastric tube, urinary catheter and drainage tube) in the chamber. The typical cases as follows. The preoperative diagnosis of a 35-year-old male patient: subarachnoid hemorrhage; skull basal fractures; left rib fracture; contused laceration in left lower lobe and hemothorax in left side; left side scapula fracture; several soft tissue injuring. His postoperative diagnosis: severe craniocerebral trauma; a. right temporal lobe laceration; b. subarachnoid hemorrhage; c. bifrontotemporal subdural effusion; d. the left temporal subdural hematomas; infection of lungs; left side scapula fracture; 3rd-10th back ribs fracture in left side; pleural effusion on both sides; multiple fractures in whole body. The following main parts of operative cooperation were included in this thesis.

\section{Methods}


All patients with varied degrees of coma on admission, and then they received conventional therapies with conventional symptomatic treatment, anti-infective methods and conventional methods of basic nursing after they entering the hospital: the conventional neurosurgical therapies of patients such as using mannitol to reduce intracranial pressure; using sensitive antibiotics to resist infection; methods of hormone therapy; nourishing brain cell and other symptomatic treatments. It is necessary to inform the patients that the pathogenic cause, the therapies and the cautions in the therapeutic process of severe craniocerebral trauma therefore improve the adherence and cooperation of patients in treatment.

All the patients need to cooperate with nursing on the basis of conventional treatment: Hyperbaric oxygen therapy. Patients treated by medical hyperbaric oxygen chamber after their surgery, the pressure of the chamber is $19.4 \mathrm{Mpa}$ with 20 minutes pressuring time and then with 25 minutes decompression time. Given oxygen once a day, each time for 1 hour and treated of 14 consecutive days (2 courses of treatment), then continued next course after 3 days interval. Comprehensive nursing intervention. Since the patients of severe craniocerebral trauma do not understand the hyperbaric oxygen chamber, it necessary to conduct healthy guidance and related psychological counseling to them, give them relevant knowledge that related the disease and the coming therapies, nurse should help patients set up their confidence to overcome the disease and improve the cooperation with treatment. At the same time, nurse should carry out specific nursing to the patients depend on their conditions: coma patient's head should be leaned on one side to avoid vomiting which caused by asphyxia; for patient with soft tissue injures should prevented the injury sites to be hurt again besides, strength their nutritional supports before the nursing; strengthening the nerve functional assessment and routine examination for patients. According the results of examinations, effectively assessing the patients' condition and taking preventions and nursing measure of complication to ensure the patients get immediate and effective treatment of systemic intervention. Guide and assist the patients to open the eustachian tube before they enter into the chamber, and in order to avoid the pressure injure the eardrums in compression stage, patients should take corresponding adjustment timely. Take care of observation in stable stage and check whether the mask for oxygen-inhalation accords with requirements. The nurse also should closely observe patients' vital signs and take emergency preparation to the patients with abnormal conditions. The nursing for the patients with tracheomy, irritability and artificial nose. Patients with tracheomy should use emergency hood. Oxygen absorbing should immediately stopped when patients appears prodromal symptoms of oxygen toxicity such as facial muscles twitching, cold sweat and phlegm when they take in oxygen in stable condition. Patients with tracheomy should be paid more attention about bleeding of the cut, the block of the tube, and the subcutaneous hematoma and emphysema. To help patients with sputum suction timely and to keep the respiratory open in package transit. To check the tightness of tracheal tube, its fixation should able to insert one finger under it, or it will oppress cervical vessel if too tight, on the contrary it will causing the removal of the tube placenta. Be caution of the pressure change in the camber that would change the volume of compartments and cause broken. After tracheotomy, the patient's trachea opening directly to the outside and the respiratory lost the filtering and nonspecific immunity protection form pathogenic microorganism in normal conditions, under the circumstances, every procedures must strictly follow the principles of aseptic technique in the treatment. Specializing the using of breathing circuits tubes and disinfecting it strictly. Replacing artificial nose everyday and disinfecting the air in the chamber before and after the treatment. Culturing the air regularly to avoid across infection. The nursing of the decompression. Strictly implementing the decompression schedule to avoid the decompression sickness in the process of decompression. The temperature of the chamber should 
reasonable controlled in compression so that to keep warm and prevent the patients from cold. Opening the drainage tubes in patients in a timely manner, preventing the injury from gas expansion during the compression. In the meanwhile, reducing the patient 's press in a slow speed and paying attention to the apostasis of patient. At the stage of reducing pressure and coming out from the chamber, the patient may have discomfort about it, nurse should explain the reasons this to patient who recovery consciousness from the treatment, and take preparation of appropriate symptomatic to this. In order to prevent the intracranial pressure appears rebound phenomenon, patients should be given dehydration, diuretics in advance, and be properly controlled the speed of transfusion. After the treatment, nurse should be careful with the patient's conditions, and they can leave without any discomfort after a break. Meanwhile, nurse should give the rehabilitation, diet and daily life direction according the recovery of patients, acquaint with their mental fluctuations and assess their mental health. The direction of discharging. Based on the recovery of patients, nurse should have a reasonable guidance to their function exercise, diet and daily life after the treatment. And inform the patients with relevant considerations after discharge. The patients should have examination and treatment immediately when they in abnormal conditions.

\section{Results}

Through the related nursing of severe craniocerebral trauma with hyperbaric oxygen therapy, the patients achieved expected surgery effect in the process and also before and after the treatment. Nurse promoted the relevant knowledge of the disease and eliminate their fears about the disease and the treatments. It improved cooperation and obtained success of the treatment and also gained the recognition from patients relatives. All those 16 patients can learn about the pathogenesis, operative treatment and the principle of hyperbaric oxygen through this period, at the same time, they were familiar with relative points for attention in treatment. Moreover, the nurse strengthened the patients with health propaganda, hyperbaric oxygen specific nursing and the directions after discharge, and took related nursing of every patient to ensure the completion of treatment. All patients actively collaborate with the staff and have achieved remarkable nursing effect.

\section{Discussion}

Severe craniocerebral trauma is a kind of disease that has high morbidity, mortality and disability rates. The state of illness changes rapidness and the survival patients suffer from different degrees of dysfunction and unable to handle their daily life, which give the heavy burden to themselves and their relatives. At present, the main clinic treatments of severe craniocerebral trauma are the conventional treatment like the combination of mannitol dehydrate with resist infection, hormone therapy and so on.

For the past few years, hyperbaric oxygen therapy applied in the severe craniocerebral trauma and gained desired results.

Hyperbaric oxygen is widely used in clinic treatment, this method guarantees the brain tissue with sufficient supply of blood and oxygen, and improve the quality of prognosis. This treatment can increase blood and oxygen quickly volume after the operation and restore normal aerobic metabolism. It improves the recovery of body and brain cells and then reduce the complications which caused by ischemia and anoxia. It also shorten the length of prognosis and enhance the quality of patients' life. And nursing care of patients during the corresponding implementation can ensure the effective oxygen absorption and effective treatments, it also improve the compliance of patients according to the controlling pressure in the chamber. For the sake of the effective ensurance 
in oxygen absorption, it is essential to keep fluency of respiratory. And sputum suction also crucial to the patients with expectoration, but the respiratory infections should be avoid, which caused by action pane. From patients admitted to this case can be seen: the patients with severe craniocerebral trauma can eliminate of symptoms and signs, and improve their living. With corresponding nursing measures in the treatment, the complications of craniocerebral trauma can be prevented and adverse reactions can reduced by high pressure. It could promote the clinical results and facilitate the early recovery of patients. However, patients with hyperbaric oxygen therapy should be closely monitored with vital signs such as blood pressure and heart rates. For the patients with abnormal conditions, the treatment should paused immediately and the emergency should prepared at once. To further improve the clinical effect, the treatment of severe craniocerebral trauma not only need nurses have good psychological quality, high professional responsibility, solid professional knowledge and skills, but also ask them have a wealth of clinic experience, acute observation and sharp observation to patients, so that to reduce perioperative morbidity and mortality.

To sum up, compared with conventional method and nursing, hyperbaric oxygen therapy is an ideal treatment method of severe craniocerebral trauma, in addition, the comprehensive nursing intervention can improve the clinical cure rate during the treatment. So it worth to be popularized and applied.

\section{References}

[1] Zhou Hongmei, Jiang Hairong. Nursing of hyperbaric oxygen therapy in patients with severe traumatic brain injury [J]. The nurse (a), 2012,03:46-48.

[2] Wang Lijun, Yan Fenghui, Long Hongchuan, Kan Qiwei. Nursing of hyperbaric oxygen therapy in patients with severe traumatic brain injury [J]. Journal of surgery, 2012,05:573-574.

[3] Xu Yuyan, Li Li, Ling Ling. Hyperbaric oxygen therapy in the treatment of severe traumatic brain injury [J]. Jilin medicine, 2011,06:1242.

[4] Li Shuwen, Tian Fu Ming, Zhou Yanxia. Nursing care of 46 patients with severe craniocerebral injury with hyperbaric oxygen therapy [J]. China Journal of medical science, 2009,11:2656-2657.

[5] $\mathrm{Wu} \mathrm{Wu}, \mathrm{Yu}$ Zhiwen. Nursing care of patients with severe craniocerebral injury treated by hyperbaric oxygen therapy [J]. Nursing and rehabilitation, 2008,03:194-195. 